\title{
The caudate nucleus and avoidance learning: A reevaluation*
}

\section{ROGER K. THOMAS and ALTON STEPHEN HILL University of Georgia, Athens, $\mathrm{Ga} .30602$}

Previous reviews suggested that caudate nucleus lesions resulted in active avoidance deficits unless the damage was small and antero-dorsally located. A reevaluation of some of the earlier studies along with the incorporation of some recent data, including data presented here for the first time, suggests that it may not be small antero-dorsal lesions that fail to reveal deficits in avoidance learning but small anterior lesions situated between the dorsal and ventral aspects of the caudate nucleus. It was further suggested that relatively low UCS intensities may have contributed to the failure to see deficits following antero-dorsal lesions.

Kirkby (1970) noted an apparent controversy about the effects of caudate nucleus lesions on active avoidance learning. The question was whether caudate lesions interfere with or have no effect on avoidance learning. Most of the works cited by Kirkby pointed to deficits, but he noted that at least three studies, including those of Albert \& Bignami (1968) and Winocur \& Mills (1969), reported no deficits. Winocur \& Mills (1970) replied to Kirkby and concluded that deficits were typically found with large postero-ventral caudate lesions but not with smaller antero-dorsal lesions. However, this conclusion may be questioned in view of a subsequent study by Neill \& Grossman (1970), who reported deficits in two-way avoidance with small antero-dorsal lesions. Albert \& Bignami (1968) had used small antero-dorsal caudate lesions but reported no deficits. Perhaps among other variables, the two studies differed on strains of rats, trials per day, and UCS intensity.

A detailed analysis of this literature reveals many differences among the studies. Differences have included species, tasks and procedures, lesion sizes and placements, and CS and UCS parameters. Deficits vs nondeficits have been reported following caudate lesions for one-way active avoidance (e.g., Mitcham \& Thomas, 1972 vs Winocur \& Mills, 1969) and two-way active avoidance (e.g., Neill \& Grossman, 1970 vs Albert \& Bignami, 1968). The present work will attempt to resolve some of the discrepancies in the rat literature. All experiments cited in this paper used rats. Kirkby (1970) and Winocur \& Mills (1970) should be consulted for related literature using animals other than rats.

We noted that the UCS intensities among caudate lesion, avoidance learning studies ranged apparently from $0.4 \mathrm{~mA}$ (Winocur \& Mills, 1969) to $3.0 \mathrm{~mA}$ (Kirkby \& Kimble, 1968). UCS intensity has been shown

\footnotetext{
*Requests for reprints should be sent to Roger $\mathrm{K}$. Thomas, Department of Psychology, University of Georgia, Athens, Ga. 30602 .
}

to be an influencing variable in avoidance studies involving nonoperated rats (e.g., Moyer \& Korn, 1964, 1966). It is interesting to note in the present context that Moyer \& Korn (1964) found that their 0.5-mA UCS resulted in significantly fewer avoidance responses in one-way avoidance training than higher UCS intensities (1.5-3.5 mA).

We attempted to replicate 'Winocur \& Mills's (1969) Experiment 1, using their UCS (0.4 mA) and two others $(0.75$ and $1.5 \mathrm{~mA})$. In Experiment 1, they trained their rats on one-way active avoidance followed by passive avoidance, where the animal was required to inhibit his learned active avoidance response. We tried to replicate their caudate lesions and their cortical control group's lesions,'but we used a nonoperated control group instead of a group comparable to their operated control group. Other differences were (a) they used a pulsating dc shock, ours was sinusoidal; (b) they used Wistar rats, we used Sprague-Dawley derived; and (c) apparently they constructed their apparatus of Plexiglas, while we used Lafayette modular units but with a Plexiglas guillotine door between the two compartments. Finally, we attempted to replicate only that part of their study which used the raising of the door dividing the apparatus plus the simultaneous onset of an overhead light as the CS. Our data are summarized in Table 1.

Like Winocur \& Mills (1969), we found no significant differences among groups with the 0.4-mA UCS. Our mean trials to criterion at this UCS intensity were comparable to theirs. As may be seen in Table 1, the caudates tended to perform more poorly than controls at the higher UCS intensities, but these differences only approached statistical significance $(F=2.85, \mathrm{df}=2 / 63$, $\mathrm{p}<.10$ ). Also, the tendency for the groups to take more trials to criterion at the $0.4-\mathrm{mA}$ UCS than at the higher UCS intensities only approached statistical significance $(\mathrm{F}=3.0, \mathrm{df}=2 / 63, \mathrm{p}<.10)$.

Unlike Winocur \& Mills (1969), we found no significant differences among the groups as a function of lesion condition in passive avoidance $(F=1.54$, $\mathrm{df}=2 / 63, \mathrm{p}<.10$ ). The differences among groups as a function of UCS intensity in passive avoidance were significant $(\mathrm{F}=6.44, \mathrm{df}=2 / 63, \mathrm{p}<.01)$. As may be seen in Table 1, this was due to the poorer performances by the groups at the 0.4-mA UCS compared to the performances of the groups at the $0.75-$ and $1.5-\mathrm{mA}$ UCS intensities. While the present work is concerned principally with active rather than passive avoidance, it may be useful to note that there appears to be agreement that caudate lesions yield passive avoidance deficits. As will be shown, we suggest that our failure to find passive avoidance deficits may have been due to inappropriately placed lesions.

Our average caudate lesion was slightly smaller and 
Table 1

Trials to Criterion in One-Way Active and Passive Avoidance in Caudate Lesioned, Neocortically Lesioned, and Nonoperated Rats as a Function of UCS Intensity

\begin{tabular}{llrrr}
\hline & & \multicolumn{3}{c}{ UCS Intensity } \\
\cline { 3 - 5 } Tasks & Groups & $.4 \mathrm{~mA}$ & $.75 \mathrm{~mA}$ & $1.5 \mathrm{~mA}$ \\
\hline \multirow{3}{*}{ Active } & Caudate & 17.5 & 16.9 & 20.4 \\
& Neocortical & 19.8 & 8.2 & 10.0 \\
& Nonoperated & 16.9 & 9.9 & 12.4 \\
& Caudate & 5.1 & 3.8 & 1.7 \\
& Neocortical & 6.3 & 2.0 & 2.5 \\
& Nonoperated & 7.8 & 4.5 & 3.6 \\
\hline
\end{tabular}

slightly more ventral than the representative lesion shown by Winocur \& Mills (1969). However, it must be noted that the ventral extent of most of our lesions was only about midway down in the caudate.

Neill \& Grossman (1970) found deficits in two-way active avoidance with dorsal and ventral anterior caudate lesions. However, they suggested that ventral lesions were more effective than dorsal lesions in producing deficits and that "dorsal lesions may be effective only if they involve the dorsolateral aspects of the head of the caudate [p. 316]." Unfortunately, our experiment was in progress when Neill and Grossman's report appeared, and our lesions turned out to be located, on the average, approximately between the two sites they suggested were most effective. A subsequent experiment from this laboratory included caudate groups with lesions that were comparable to Neill and Grossman's ventral group, and significant deficits were found in one- and two-way active avoidance and passive avoidance (Mitcham \& Thomas, 1972). The one-way active avoidance procedure was the same as Winocur \& Mills's (1969), except that a 0.75-mA UCS was used.

In light of Neill \& Grossman's (1970) suggestions concerning dorsolateral and ventral anterior caudate lesions and our data (Table 1) based on a lesion located approximately between their recommended sites, it may be useful to reconsider the caudate lesions in Albert \& Bignami's (1968) experiment; it should be recalled that they did not find deficits in two-way avoidance following caudate lesions. Their illustrated typical caudate lesion appears very similar to the average lesion in our experiment. Thus, it is suggested that small anterior caudate lesions which are located between the dorsal and ventral aspects of the nucleus may be minimally effective in producing deficits in avoidance learning. That the caudate nucleus may be comprised of separable functional regions has been suggested and documented by Divac $(1968,1972)$.

It is suggested that an inappropriately placed caudate lesion accounts for the failure to see deficits in Albert \& Bignami's (1968) study and our study summarized here. Contrary to previous suggestion (Winocur \& Mills, 1970), it is not a small anterior dorsal caudate lesion that is deemed to be inappropriate but an anterior lesion that is located between the dorsal and ventral aspects of the caudate nucleus in the rat. Based on a consideration of Albert \& Bignami's (1968), Neill \& Grossman's (1970), and our lesions, it is suggested that suitable coordinates to demonstrate this noneffective lesion are $2.2 \mathrm{~mm}$ anterior and $3.5 \mathrm{~mm}$ lateral to bregma and $5.0 \mathrm{~mm}$ down from the surface of the dura. It is suggested that the anterior coordinates may range, at least, from $1.8 \mathrm{~mm}$ (approximately Neill and Grossman's coordinate) to $3.5 \mathrm{~mm}$ (Albert and Bignami's coordinate; both coordinates re bregma). It is further suggested that Winocur \& Mills's (1969) UCS intensity was insufficient to reveal deficits that might have been evident in their caudate lesioned animals had they used higher UCS intensities.

A study by Kirkby (1969) needs to be considered in the context of these conclusions. Kirkby compared caudate lesioned, cortically lesioned, and nonoperated rats on several measures. One measure was a brightness discrimination in a Y maze. To avoid footshock, the rat had to enter the lighted stem (randomly alternated) of the $\mathrm{Y}$ within $10 \mathrm{sec}$. Failure to enter the correct alley was followed by $10 \mathrm{sec}$ of possible exposure to $0.6-\mathrm{mA}$ shock, and failure beyond that time resulted in $10 \mathrm{sec}$ possible exposure to 1.2-mA shock. The groups did not differ on this active avoidance response [a fact not mentioned in Kirkby's (1970) assessment of the caudate lesions, active avoidance literature]. The representative lesion shown was sufficiently large and anterior to constitute a possible exception to the conclusions suggested in this paper. Three explanations for the lack of a deficit by the caudate lesioned rats on this measure in Kirkby's study (1969) may be suggested. (1) The lesion shown minimally invades the ventral region (compare with Neill \& Grossman, 1970; Mitcham \& Thomas, 1972) and the lesion on the left may not be sufficiently dorsolateral, in view of Neill \& Grossman's (1970) suggestion, to be effective. (2) The 0.6-mA shock may have produced the correct response most of the time, precluding exposure to the $1.2 \mathrm{~mA}$ shock, and the lower value may not have been sufficient to reveal differences among the groups. (3) The criterion measures for learning-five correct responses in one six-trial session-may have been inadequate (Grant, 1947) to conclude that the rats had learned the brightness discrimination. Had a more stringent criterion been used, the caudate lesioned group might have taken significantly more trials to criterion.

Obviously, further research is needed to settle the question of the involvement of the caudate nucleus in avoidance learning. Two testable hypotheses have been suggested here, namely, that lesion placement and UCS intensity are important variables. Hopefully, future researchers in this area will be more critical of their procedures and will be more concerned with the comparability of their measures and those previously used. 


\section{REFERENCES}

Albert, M., \& Bignami, G. Effects of frontal, median cortical and caudate lesions on two-way avoidance learning by rats. Physiology \& Behavior, 1968, 3, 141-147.

Divac, I. Functions of the caudate nucleus. Acta Biologiae Experimentalis, 1968, 28, 107-120.

Divac, I. Neostriatum and functions of prefrontal cortex. Acta Neurobiologiae Experimentalis, 1972, 32, 461-477.

Grant, D. A. Additional tables of the probability of "runs" of correct responses in learning and problem-solving. Psychological Bulletin, 1947, 44, 276-279.

Kirkby, R. J. Caudate nucleus lesions and perseverative behavior. Physiology \& Behavior, 1969, 4, 451-454.

Kirkby, R. J. The caudate nucleus and active avoidance: $A$ comment on the report of Winocur and Mills. Psychonomic Science, 1970, 18, 269.

Kirkby, R. J., \& Kimble, D. P. Avoidance and escape behavior following striatal lesions in the rat. Experimental Neurology, $1968,20,215-227$.

Mitcham, J. C., \& Thomas, R. K., Jr. Effects of substantia nigra and caudate nucleus lesions on avoidance learning in rats.
Journal of Comparative \& Physiological Psychology, 1972, 81, 101-107.

Moyer, K. E., \& Korn, J. H. Effect of UCS intensity on the acquisition and extinction of an avoidance response. Journal of Experimental Psychology, 1964, 67, 352-359.

Moyer, K. E., \& Korn, J. H. Effect of UCS intensity on the acquisition and extinction of a one-way avoidance response. Psychonomic Science, 1966, 4, 121-122.

Neill, D. B., \& Grossman, S. P. Behavioral effects of lesions or cholinergic blockade of the dorsal and ventral caudate of rats. Journal of Comparative \& Physiological Psychology, 1970, 71, 311-317.

Winocur, G, \& Mills, J, A Effects of caudate lesions on avoidance behavior in rats. Journal of Comparative \& Physiological Psychology, 1969, 68, 552-557.

Winocur, G., \& Mills, J. A. The caudate nucleus and active avoidance: Reply to Kirkby. Psychonomic Science, 1970, 18, 270.

(Received for publication February 27, 1973.) 